\title{
Mitgliederverzeichnis/Liste des membres
}

Präsident/Président: P.D. Dr. Hans Deucher, Kantonsspital Aarau; Vizepräsident/ VicePrésident: P.D. Dr. H. Hotz, Haldenstrasse 37a, Luzern; Aktuar/Greffier: Dr. G. Miller, Hauptgasse 58, Solothurn; Beisitzer/Assesseurs: Prof. G. Candardjis, Institut universitaire de Radiologie, Lausanne; Prof. J.-C. Rudler, Clinique chir., Hôpital Cantonal, Geneve;

Zentralsekretär/Secretaire general: Dr. E. Hafter, Tödistrasse 36, Zurich.

1964

I. Ehrentnítglíeder

Bansi, H. W., Prof. Berg, H. H., Prof.

Caroli, Jacques, Prof. Debray, Charles, Prof. Feissly, Robert, P.D. Froehlich, Albert Gutmann, René-A. Henning, Norbert, Prof. Hepp, Jacques Hunt, Thomas Kalk, H., Prof. Lambling, Andre, Prof. Paschoud, Henri, P. D. Mogena, Heliodoro-G., Prof. Santy, Paul, Prof. Verzar, F., Prof.

Bonnet, Georges Coppo, Prof. Ghione, U., Prof. Martini, G. A., Prof. Demeulenaere, Léo Massion, Joseph Menghini, Prof.

II. Korrespondíerend $\beta$ Mítglíeder

15 , rue Nicolas-Larbaud Vichy

Clinica Med. Univ. Modena

15, via Nuccio di Buoninsegna Milano

Mediz. Universitätsklinik Marburg/Lahn

Faculté de méd., Univ. Gand, Belgique

148, bd Brandt-Whitlock Bruxelles

Ospedale Civile Macerata

Mitgliederverzeichnis/Liste des membres

303

Porcher, Pierre Rathcke, L., Prof. Rissel, E., Prof. Taylor, Hermon Vachon, A., Prof.

Vandenbroucke, J., Prof.

Viguié, Roger Villardel, Jaquinto

Hôpital St-Antoine

Kreis-Krankenhaus

Rathausstraße 16

London-Hospital

1, quai Général-Sarrail

Klinik voor inwendige

Geneeskunde

79, bd Malesherbes 
Bruch 125

Paris

Ludwigsburg

WienI

London

Lyon

Leuven, Belgien

Paris VIIIe Barcelona

1935 bis 19371938 bis 19401941 bis 19431944 bis 19461947 bis 19491950 bis 19521953 bis 19551956 bis 19581959 bis 19601961 bis 19621963 bis 1964

III. Ehemalige Präsidenten

Feissly, Robert, P.D. Lausanne

Stalder, Hans $\dagger \quad$ Basel

Saloz, Charles $\dagger \quad$ Geneve

Löffler, Wilhelm, Prof. Zurich

Paschoud, Henri, P. D. Lausanne

Saegesser, Max, Prof. Bern

Demole, Michel, Prof.Geneve

Kapp, Hermann, P.D. Basel

Hafter, Ernst Zurich

Candardjis, G., Prof. Lausanne

Deucher, Franz, P. D. Aarau

IV. Aktívmítglíeder

A. Internisten, praktísche Arzte

Ackermann, William-R. AfFolter, Heinz Alphonse, Pierre Ammann, Rudolf

Antognini, Guido Audeoud, Roger Balissat, Edouard Barandun, Silvio Berger, Erwin, Prof.

Bickel, Georges, Prof. Binswanger, Dieter Boitel, Yves Borer, Fernand Borsati, Giampiero

Bubb, Werner Bullet, Francois Buser, M. Clémençon, Gerard

304

Mitgliederverzeichnis/Liste des membres

Demole, Michel, Prof.

Du Bois, Georges-A.

Dufour, Jean-Jacques

Eisner, Wolf

Elmiger, Franz

Fahrländer, Hansjürg, P. D.

Fatzer, Guy

Fierz, Felix

Frick, Paul, Prof.

Funk, Paul

Gassmann, R.

Gätzi, Walter

Grogg, Edwin 
Gross, Cami Пe

Guckelberger, Martin, Prof.

Haemmerli, Armin

Haemmerli, Urs

Hafter, Ernst

Hefti, Max Lionel

Hegglin, Robert, Prof.

Hofstetter, Jean-René, P. D.

Hotz, Heinrich, P. D.

Huber, Konrad

Huber, Walter

Jaccard, Gaston, P. D.

Jacot, Claude

Jasinski, B.

Jeanneret, Pierre

Jordi, Alfred

Junet, Robert, P. D.

Kapp, Hermann, Prof.

Karrer, Jürg

Koller, Fritz, Prof.

Kopp, Eugen

Krieg, Pierre

Kunz, Robert, Dr. chem.

Kym, Otto Laemmli, Karl Leutenegger, Friedrich Loizeau, Edouard Mach, Rene, Prof.

Magnenat, Pierre Mamie, Maurice Mangold, Renward Marko£f, Nicola, P.D. Martin, Eric, Prof.

Mitgliederverzeichnis/Liste des membres

306 Mitgliederverzeichnis/Liste des membres

B. Chirurgen

Berchtold, Rudolf, P.D. Blöchlinger, Louis Buffat, Jean-David, P.D. Bühlmann, Eric Calame, Andre, Prof. Chanal, Georges, P.D. Chaperon, Louis Coquoz, Michel Deucher, Franz, P.D.

Eckmann, Leo, P.D. Fink, Robert Francioli, Pierre, P.D. Gardaz, Emile Häberlin, Paul Hess, Walter, Prof. Howald, Hans Juillard, Edouard Kaiser, Ernst Keller, Andre Lehner, August Montant, Robert, Prof. Müller, Charles-André Naef, Andreas-P. Naz, Edouard Nicole, Robert, Prof. Nietlispach, Lukas Nissen, Rudolf, Prof. Pettavel, Jacques Piotet, Gustave Renfer, Eugen Rollier, Jean

Rudler, Jean-Claude, Prof. Ryncki, Pierre V. Saegesser, Francois, Prof. Saegesser, Max, Prof. Schobinger, A. Willenegger, Hans, Prof.

Bürgerspital Bubenbergplatz 5 38, avenue Rumine Laupenstraße 6 12, route Florissant 6, rue Ch.-Bonnet

16, rue du Simplon Chir. Abt., Kantonsspital Schildknechtstraße 30 Krankenhaus 42, avenue de Rumine 8, Grand-Pont Bleicherweg 52 Limmatquai 122 Limmatquai 30

2, rue des Tilleuls

Stadtspital 
Route d'Yverdon

Sonnenhof 4

18 , Corraterie

16, ch. de Mallieux

48, avenue de Rumine

3, place Claparède

Socinstraße 22

Kantonsspital

Spitalstraße 21

Le Planier, ch. Val-d'or 22, route St-Cergues Wabernstraße 22

Clin. chir., Hôpital cantonal 1, galeries Benjamin-Constant 31, avenue de Rumine Schänzlihalde

23 Kreuzmattweg 7 Kantonsspital

Solothurn

Bern

Lausanne

Bern

Geneve

Geneve

Châtel-St-Denis

Vevey

Aarau

Bern

Horgen

Lausanne

Lausanne

Zurich 2

Zurich 1

Zurich 1

Porrentruy

Zurich 10/37

Payerne

Luzern

Geneve

Pully, Lausanne

Lausanne

Geneve

Basel

Aarau

Basel

Pully/VD

Nyon

Bern

Savièse/VS

Geneve

Lausanne

Lausanne 
Bern

Luzern

Liestal/BL

C. Radíologen

Bivetti, Jürg Bugnion, Michel Candardjis, Georges, Prof. Deucher, Walter Etter, Hans Ganz, Ernst

Talstraße 65 Zurich 1

6, avenue Georgette Lausanne

Inst. univ. de Radiologie Lausanne

Stadelhoferstraße 42 Zurich 1

Kantonsspital Luzern

Bahnhofstraße $61 \quad$ Zurich 1

Mitgliederverzeichnis/Liste des membres

Güntert, Walter

Kleinert, Robert Kollbrunner, Felix Pasquier, Jean Ramseyer, Marc Renfer, Hansrudolf Schärer,

Kurt

Thommen, Bruno

Wieser, Constant

Wellauer, Josef, Prof. Zimmer, Emile, Prof.

Alder, Albert, Prof. Charbonnier, Albert Choisy, Robert Decker, Pierre, Prof. Gigon, Alfred,

Prof. Heusser, Heinrich, Prof. Löffler, W., Prof. Müller, Hermann

22 Gastroenterologia Vol. 101, No. 5 (1964) 